\title{
MODULE STRUCTURE OF CELLS IN UNEQUAL-PARAMETER HECKE ALGEBRAS
}

\author{
THOMAS PIETRAHO
}

\begin{abstract}
A conjecture of Bonnafé, Geck, Iancu, and Lam parametrizes Kazhdan-Lusztig left cells for unequal-parameter Hecke algebras in type $B_{n}$ by families of standard domino tableaux of arbitrary rank. Relying on a family of properties outlined by Lusztig and the recent work of Bonnafé, we verify the conjecture and describe the structure of each cell as a module for the underlying Weyl group.
\end{abstract}

\section{$\S 1$. Introduction}

Consider a Coxeter system $(W, S)$, a positive weight function $L$, and the corresponding generic Iwahori-Hecke algebra $\mathcal{H}$. As detailed by Lusztig in [21], a choice of weight function gives rise to a partition of $W$ into left, right, and two-sided Kazhdan-Lusztig cells, each of which carries the structure of an $\mathcal{H}$-module as well as a representation of $W$. The cell decomposition of $W$ is understood for all finite Coxeter groups and all choices of weight functions with the exception of type $B_{n}$. Focusing on this remaining case, we write $W=W_{n}$. A weight function is then specified by a choice of two integer parameters $a$ and $b$ assigned to the simple reflections in $W_{n}$ :

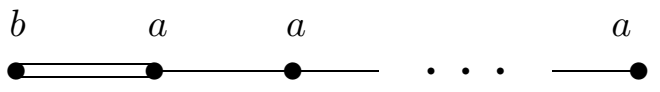

Given $a, b \neq 0$, write $s=b / a$ for their quotient. We have the following description of cells due to Bonnafé, Geck, Iancu, and Lam. It is stated in terms of a family of generalized Robinson-Schensted algorithms $G_{r}$ which define bijections between $W_{n}$ and same-shape pairs of domino tableaux of rank $r$.

Conjecture. ([3]) Consider a Weyl group $W_{n}$ of type $B_{n}$ with a weight function $L$ and parameter $s$ defined as above.

Received February 19, 2009. Accepted August 10, 2009.

2000 Mathematics Subject Classification. 20C08, 05E10.

(C) 2010 by The Editorial Board of the Nagoya Mathematical Journal 
(1) When $s \notin \mathbb{N}$, let $r=\lfloor s\rfloor$. Two elements of $W_{n}$ lie in the same KazhdanLusztig left cell whenever they share the same right tableau in the image of $G_{r}$.

(2) When $s \in \mathbb{N}$, let $r=s-1$. Two elements of $W_{n}$ lie in the same KazhdanLusztig left cell whenever their right tableaux in the image of $G_{r}$ are related by moving through a set of noncore open cycles.

Significant progress has been made in the verification of the above, which is detailed in Section 3.3. Most recently, Bonnafé has shown that if a certain family of statements conjectured by Lusztig is assumed to hold, then the conjecture itself holds if $s \notin \mathbb{N}$; furthermore, if $s \in \mathbb{N}$, then Kazhdan-Lusztig left cells are unions of the sets described above (see [2]). We sharpen this result and verify that the conjecture holds in the latter case as well.

We concurrently describe the representations carried by Kazhdan-Lusztig left cells. The canonical parameter set for the irreducible characters of $W_{n}$ consists of ordered pairs of partitions $(d, f)$ where the parts of $d$ and $f$ sum to $n$. As detailed in Section 4.1, there is a natural identification of this parameter set with the set of partitions $\mathcal{P}_{r}(n)$ of a fixed rank $r$. Since $\mathcal{P}_{r}(n)$ corresponds exactly to the shapes of rank $r$ domino tableaux, the parametrization of Kazhdan-Lusztig left cells via standard tableaux of fixed rank in the above conjecture suggests which representation should be carried by each cell for every choice of weight function. Mainly, the irreducible constituents of the representation carried by each cell should correspond to the shapes of the rank $r$ tableaux of its elements, with $r$ determined from the parameter $s$ as in the above conjecture. We verify that this suggested representation is indeed the one carried by each cell.

The approach here is based on Geck's characterization of left cells as constructible representations, that is, those representations which are obtained by successive truncated parabolic induction and tensoring with the sign representation (see [9]). Section 2 details the general construction of KazhdanLusztig cells in an unequal-parameter Hecke algebra and extends a result of Lusztig on the intersection of left and right cells to the unequal-parameter setting. Section 3 details the situation in type $B_{n}$ and the relevant combinatorics. Section 4 examines constructible representations and provides a combinatorial description of truncated parabolic induction and tensoring with sign, mimicking the work of McGovern [22] in the equal-parameter case. The final section contains the proof of the main results. 


\section{§2. Unequal-parameter Hecke algebras}

To begin, a brief recounting of the definitions of unequal-parameter Hecke algebras and the corresponding Kazhdan-Lusztig cells is given, following $[21]$.

\subsection{Kazhdan-Lusztig cells}

Consider a Coxeter system $(W, S)$, and let $\ell$ be the usual length function. A weight function $L: W \rightarrow \mathbb{Z}$ satisfies $L(x y)=L(x)+L(y)$ whenever $\ell(x y)=\ell(x)+\ell(y)$ and is uniquely determined by its values on $S$. We will consider those weight functions which take positive values on all $s \in S$.

Let $\mathcal{H}$ be the generic Iwahori-Hecke algebra over $\mathcal{A}=\mathbb{Z}\left[v, v^{-1}\right]$ with parameters $\left\{v_{s} \mid s \in S\right\}$, where $v_{x}=v^{L(x)}$ for all $x \in W$. The algebra $\mathcal{H}$ is free over $\mathcal{A}$ and has a basis $\left\{T_{x} \mid x \in W\right\}$. Multiplication in $\mathcal{H}$ takes the form

$$
T_{s} T_{x}= \begin{cases}T_{s x} & \text { if } \ell(s x)>\ell(x), \\ T_{s x}+\left(v_{s}-v_{s}^{-1}\right) T_{x} & \text { if } \ell(s x)<\ell(x) .\end{cases}
$$

As in [21, Section 5.2], it is possible to construct a Kazhdan-Lusztig basis of $\mathcal{H}$, which we denote by $\left\{C_{x} \mid x \in W\right\}$. In terms of it, multiplication takes the form

$$
C_{x} C_{y}=\sum_{z \in W} h_{x y z} C_{z}
$$

for some $h_{x y z} \in \mathcal{A}$. Although we suppress it in the notation, all of these notions depend on the specific choice of the weight function $L$.

Definition 2.1. Fix $(W, S)$, a Coxeter system with a weight function $L$. We will write $y \leq_{\mathcal{L}} x$ if there exists $s \in S$ such that $C_{y}$ appears with a nonzero coefficient in $C_{s} C_{x}$. By taking the transitive closure, this binary relation defines a preorder on $W$, which we also denote by $\leq_{\mathcal{L}}$. Let $y \leq_{\mathcal{R}} x$ if and only if $y^{-1} \leq_{\mathcal{L}} x^{-1}$, and define $\leq_{\mathcal{L R}}$ as the preorder generated by $\leq_{\mathcal{L}}$ and $\leq_{\mathcal{R}}$.

Each of the above preorders defines equivalence relations which we denote by $\sim_{\mathcal{L}}, \sim_{\mathcal{R}}$, and $\sim_{\mathcal{L} \mathcal{R}}$, respectively. The resulting equivalence classes are called the left, right, and two-sided Kazhdan-Lusztig cells of $W$. As described in [21, Section 8.3], each Kazhdan-Lusztig cell gives rise to a representation of $\mathcal{H}$. If $\mathfrak{C}$ is a Kazhdan-Lusztig left cell and $x \in \mathfrak{C}$, then define

$$
[\mathfrak{C}]_{\mathcal{A}}=\bigoplus_{y \leq \mathcal{L}^{x}} \mathcal{A} C_{y} / \bigoplus_{y \leq \mathcal{L}^{x}, y \notin \mathfrak{C}} \mathcal{A} C_{y}
$$


This is a quotient of two left ideals in $\mathcal{H}$ and, consequently, is itself a left $\mathcal{H}$-module; it does not depend on the specific choice of $x \in \mathfrak{C}$, is free over $\mathcal{A}$, and has a basis $\left\{e_{x} \mid x \in \mathfrak{C}\right\}$ indexed by elements of $\mathfrak{C}$ with $e_{x}$ the image of $C_{x}$ in the above quotient. The action of $\mathcal{H}$ on $[\mathfrak{C}]_{\mathcal{A}}$ is determined by

$$
C_{x} e_{y}=\sum_{z \in \mathfrak{C}} h_{x y z} e_{z}
$$

for $x \in W$ and $y \in \mathfrak{C}$. Via the specialization $\left(v_{s} \mapsto 1\right)$ for $s \in S$, this construction gives rise to an $\left(\mathcal{H}_{\mathbb{C}}=\mathbb{C}[W]\right)$-module, which we denote $[\mathfrak{C}]$. Note that, in general, we cannot identify $[\mathfrak{C}]_{\mathcal{A}}$ with $[\mathfrak{C}]$ directly. However, if we let $K$ be the fraction field of the ring $\mathcal{A}$ and define $\mathcal{H}_{K}=K \otimes_{\mathcal{A}} \mathcal{H}$, then by $[15$, Sections 8.1.7 and 9.3.5], we can identify $\mathcal{H}_{K}$-modules and the representations of $W$. In what follows, we refer to $\mathbb{C}[W]$-modules simply as $W$-modules and to $[\mathfrak{C}]$ as the representation of $W$ carried by $\mathfrak{C}$. A similar construction can be used to define representations carried by the right and two-sided cells of $W$.

\subsection{A family of properties}

The main results of this paper rely on a family of conjectures formulated by Lusztig in [21, Section 14]. In the equal-parameter case, that is, when $L$ is a multiple of the length function $\ell$, a number of results about KazhdanLusztig cells depend on positivity results derived by geometric methods of intersection cohomology. Unfortunately, this positivity does not hold for unequal-parameter Hecke algebras (for examples, see [20, Section 6], [10, Section 2.7]). As a substitute, Lusztig detailed a list of properties which both axiomatize known equal-parameter results and outline methods of approaching nonpositivity in general.

In order to list Lusztig's conjectures, we must first define two integervalued functions on $W$. For any $z \in W$, let $\boldsymbol{a}(z)$ be the smallest nonnegative integer so that $h_{x y z} \in v^{a(z)} \mathbb{Z}\left[v^{-1}\right]$ for every $x$ and $y$ in $W$, and write $\gamma_{x y z^{-1}}$ for the constant term of $v^{-\boldsymbol{a}(z)} h_{x y z}$. If $p_{x y}$ is defined by $C_{y}=\sum_{x \in W} p_{x y} T_{x}$, then $\left[21\right.$, Section 5.4] shows that $p_{1 z}$ is nonzero. We write

$$
p_{1 z}=n_{z} v^{-\Delta(z)}+\text { terms of smaller degree in } v
$$

thereby defining a constant $n_{z}$ and integer $\Delta(z)$ for every $z \in W$. Finally, let

$$
\mathcal{D}=\{z \in W \mid \boldsymbol{a}(z)=\Delta(z)\}
$$


Lusztig has conjectured that the following statements are true in the general setting of unequal-parameter Hecke algebras.

P1. For any $z \in W$, we have $\boldsymbol{a}(z) \leq \Delta(z)$.

P2. If $d \in \mathcal{D}$ and $x, y \in W$ satisfy $\gamma_{x, y, d} \neq 0$, then $x=y^{-1}$.

P3. If $y \in W$, there exists a unique $d \in \mathcal{D}$ such that $\gamma_{y^{-1}, y, d} \neq 0$.

P4. If $z^{\prime} \leq_{\mathcal{L R}} z$, then $\boldsymbol{a}\left(z^{\prime}\right) \geq \boldsymbol{a}(z)$. Hence, if $z^{\prime} \sim_{\mathcal{L R}} z$, then $\boldsymbol{a}(z)=\boldsymbol{a}\left(z^{\prime}\right)$.

P5. If $d \in \mathcal{D}, y \in W$, and $\gamma_{y^{-1}, y, d} \neq 0$, then $\gamma_{y^{-1}, y, d}=n_{d}= \pm 1$.

P6. If $d \in \mathcal{D}$, then $d^{2}=1$.

P7. For any $x, y, z \in W$, we have $\gamma_{x, y, z}=\gamma_{y, z, x}$.

P8. Let $x, y, z \in W$ be such that $\gamma_{x, y, z} \neq 0$. Then $x \sim_{\mathcal{L}} y^{-1}, y \sim_{\mathcal{L}} z^{-1}$, and $z \sim \mathcal{L} x^{-1}$.

P9. If $z^{\prime} \leq_{\mathcal{L}} z$ and $\boldsymbol{a}\left(z^{\prime}\right)=\boldsymbol{a}(z)$, then $z^{\prime} \sim_{\mathcal{L}} z$.

P10. If $z^{\prime} \leq_{\mathcal{R}} z$ and $\boldsymbol{a}\left(z^{\prime}\right)=\boldsymbol{a}(z)$, then $z^{\prime} \sim_{\mathcal{R}} z$.

P11. If $z^{\prime} \leq_{\mathcal{L R}} z$ and $\boldsymbol{a}\left(z^{\prime}\right)=\boldsymbol{a}(z)$, then $z^{\prime} \sim_{\mathcal{L R}} z$.

P12. Let $I \subseteq S$ and $W_{I}$ be the parabolic subgroup defined by $I$. If $y \in W_{I}$, then $\boldsymbol{a}(y)$ computed in terms of $W_{I}$ is equal to $\boldsymbol{a}(y)$ computed in terms of $W$.

P13. Any left cell $\mathfrak{C}$ of $W$ contains a unique element $d \in \mathcal{D}$. We have $\gamma_{x^{-1}, x, d} \neq 0$ for all $x \in \mathfrak{C}$.

P14. For any $z \in W$, we have $z \sim \mathcal{L R} z^{-1}$.

P15. If $v^{\prime}$ is an indeterminate and $h_{x y z}^{\prime}$ is obtained from $h_{x y z}$ via the substitution $v \mapsto v^{\prime}$, then whenever $\boldsymbol{a}(w)=\boldsymbol{a}(y)$, we have

$$
\sum_{y^{\prime}} h_{w x^{\prime} y^{\prime}}^{\prime} h_{x y^{\prime} y}=\sum_{y^{\prime}} h_{x w y^{\prime}} h_{y^{\prime} x^{\prime} y}^{\prime}
$$

The statements P1-P15 are known to hold for finite Weyl groups in the equal-parameter case by Kazhdan and Lusztig [17] and Springer [26]. If the Coxeter system is of type $I_{2}(m), H_{3}$, or $H_{4}$, they follow from Alvis [1] and DuCloux [5]. In the unequal-parameter case, $\mathbf{P 1 - P 1 5}$ have been verified in types $I_{2}(m)$ and $F_{4}$ by Geck [13] and in the so-called asymptotic case of type $B_{n}$ by Geck and Iancu [14] and Geck [12], [13]. Although the geometric approach from which the above follow in the equal-parameter case is not available in the general unequal-parameter case, it seems that it may not be required. At least in type $A$, Geck [11] has shown that P1-P15 hold via elementary, purely algebraic methods. 


\subsection{The asymptotic ring $J$}

The goal of this section is to verify [19, Lemma 12.15] in our more general setting. It begins with a brief discussion of Lusztig's ring $J$, which can be viewed as an asymptotic version of $\mathcal{H}$. Although originally defined in the equal-parameter case, its construction also makes sense in the setting of unequal-parameter Hecke algebras under the assumption that the conjectures P1-P15 hold. Using the methods developed in [21], $J$ provides a way of studying the left-cell representations of $\mathcal{H}$.

Recall the integers $\gamma_{x y z}$ defined for all $x, y$, and $z$ in $W$ as the constant terms of $v^{a(z)} h_{x y z^{-1}}$. Then $J$ is the free abelian group with basis $\left\{t_{x} \mid x \in\right.$ $W\}$. To endow it with a ring structure, define a bilinear product on $J$ by

$$
t_{x} \cdot t_{y}=\sum_{z \in W} \gamma_{x y z} t_{z^{-1}}
$$

for $x$ and $y$ in $W$. Conjectures P1-P15 allow us to state the following results.

Theorem 2.2. ([21]) Assuming conjectures P1-P15, the following hold:

(1) $J$ is an associative ring with identity element $1_{J}=\sum_{d \in \mathcal{D}} n_{d} t_{d}$;

(2) the group algebra $\mathbb{C}[W]$ is isomorphic as a $\mathbb{C}$-algebra to $J_{\mathbb{C}}=\mathbb{C} \otimes_{\mathbb{Z}} J$.

Following [21, Section 20.2], we will write $E_{\boldsymbol{A}}$ for the $J_{\mathbb{C} \text {-module corre- }}$ sponding to a $\mathbb{C}[W]$-module $E$. It shares its underlying space with $E$, while the action of an element of $J_{\mathbb{C}}$ is defined by the action of its image under the isomorphism with $\mathbb{C}[W]$. Consider a left cell $\mathfrak{C}$ of $W$, and define $J_{\mathbb{C}}^{\mathfrak{C}}$ to be $\bigoplus_{x \in \mathfrak{C}} \mathbb{C} t_{x}$. By P8, this is a left ideal in $J_{\mathbb{C}}$. Furthermore, we have the following.

Theorem 2.3. ([21]) Assuming that the conjectures P1-P15 hold, the $J_{\mathbb{C}}$-modules $J_{\mathbb{C}}^{\mathfrak{C}}$ and $[\mathfrak{C}]_{\text {a }}$ are isomorphic.

We are ready to address [19, Lemma 12.15]. Its original proof relies on a characterization of left cells in terms of the dual bases $\left\{C_{x}\right\}$ and $\left\{D_{x}\right\}$ stated in [19, index item 5.1.14]. This result in turn relies on positivity properties which do not hold in the unequal-parameter case, and, therefore, a new approach to the lemma is required. The idea of using $J$ in the present proof is due to Geck.

Lemma 2.4. Assume that conjectures $\mathbf{P} 1-\mathbf{P} 15$ hold. If $\mathfrak{C}$ and $\mathfrak{C}^{\prime}$ are two left cells in $W$ with respect to a weight function $L$, then

$$
\operatorname{dim} \operatorname{Hom}_{W}\left([\mathfrak{C}],\left[\mathfrak{C}^{\prime}\right]\right)=\left|\mathfrak{C} \cap \mathfrak{C}^{\prime-1}\right| .
$$


Proof. Let $x \in \mathfrak{C}^{-1} \cap \mathfrak{C}^{\prime}$, and define a map $\phi_{x}$ on $J_{\mathbb{C}}^{\mathfrak{C}}$ via $\phi_{x}\left(t_{y}\right)=t_{y} t_{x}$. With $x$ and $y$ as above, we can write

$$
t_{y} t_{x}=\sum \gamma_{y x z} t_{z^{-1}}
$$

For $\gamma_{y x z} \neq 0, \mathbf{P} 8$ implies that $x \sim_{\mathcal{L}} z^{-1}$. Since $x \in \mathfrak{C}^{\prime}$, this forces $t_{y} t_{x}$ to lie in $J_{\mathbb{C}}^{\mathfrak{C}^{\prime}}$, and we have in fact defined a map $\phi_{x}: J_{\mathbb{C}}^{\mathfrak{C}} \rightarrow J_{\mathbb{C}}^{\mathfrak{C}^{\prime}}$.

As $x$ runs over the set $\mathfrak{C}^{-1} \cap \mathfrak{C}^{\prime}$, the maps $\phi_{x}$ are linearly independent. Assume that, for some constants $a_{x}$, we have

$$
\sum_{x \in \mathfrak{C}^{-1} \cap \mathfrak{C}^{\prime}} a_{x} \phi_{x}=0
$$

and, consequently,

$$
\sum_{x \in \mathfrak{C}^{-1} \cap \mathfrak{C}^{\prime}} a_{x} t_{y} t_{x}=0
$$

for all $y \in \mathfrak{C}$. In particular, if $d$ is the unique element in $\mathcal{D} \cap \mathfrak{C}$ guaranteed by $\mathbf{P} \mathbf{1 3}$, then we also have

$$
\sum_{x \in \mathfrak{C}^{-1} \cap \mathfrak{C}^{\prime}} a_{x} t_{d} t_{x}=\sum_{x \in \mathfrak{C}^{-1} \cap \mathfrak{C}^{\prime}} \pm a_{x} t_{x}=0
$$

where the first equality follows from P2, P5, P7, and P13. But this means that $a_{x}=0$ for all relevant $x$ or, in other words, that the $\phi_{x}$ are linearly independent. We can therefore conclude that $\operatorname{dim} \operatorname{Hom}_{J_{\mathbb{C}}}\left(J_{\mathbb{C}}^{\mathfrak{C}}, J_{\mathbb{C}}^{\mathfrak{C}^{\prime}}\right) \geq\left|\mathfrak{C}^{-1} \cap \mathfrak{C}^{\prime}\right|$. Since this inequality is true for all pairs of left cells $\mathfrak{C}$ and $\mathfrak{C}^{\prime}$ in $W$, we have

$$
\sum_{\mathfrak{C}, \mathfrak{C}^{\prime}} \operatorname{dim} \operatorname{Hom}_{J_{\mathbb{C}}}\left(J_{\mathbb{C}}^{\mathfrak{C}}, J_{\mathbb{C}}^{\mathfrak{C}^{\prime}}\right) \geq \sum_{\mathfrak{C}, \mathfrak{C}^{\prime}}\left|\mathfrak{C}^{-1} \cap \mathfrak{C}^{\prime}\right| .
$$

The right side of this inequality is just the order of $W$ since each of its elements lies in a unique left and a unique right cell. On the other hand, by the correspondence resulting from Theorem 2.3, the left side is

$$
\operatorname{dim} \operatorname{Hom}_{J_{\mathbb{C}}}\left(\sum_{\mathfrak{C}} J_{\mathbb{C}}^{\mathfrak{C}}, \sum_{\mathfrak{C}^{\prime}} J_{\mathbb{C}}^{\mathfrak{C}^{\prime}}\right)=\operatorname{dim} \operatorname{Hom}_{W}\left(\operatorname{Reg}_{W}, \operatorname{Reg}_{W}\right)=|W| .
$$

Hence the original inequality must be in fact an equality, and the lemma follows.

We immediately obtain the following corollary, whose proof is identical to that of [19, Corollary 12.17].

Corollary 2.5. Assume that conjectures P1-P15 hold and that the representations carried by the left cells of $W$ with respect to a weight function $L$ are multiplicity-free. Then $\mathfrak{C} \cap \mathfrak{C}^{-1}$ is the set of involutions in $\mathfrak{C}$. 


\section{$\S 3$. Type $B_{n}$}

The goal of this section is to detail the combinatorics of arbitrary-rank standard domino tableaux necessary to describe Kazhdan-Lusztig cells in type $B_{n}$.

\subsection{Domino tableaux}

Consider a partition $p$ of a natural number $n$. We will view it as a Young diagram $Y_{p}$, a left-justified array of squares whose row lengths decrease weakly. The square in row $i$ and column $j$ of $Y_{p}$ will be denoted $s_{i j}$, and a pair of squares in $Y_{p}$ of the form $\left\{s_{i j}, s_{i+1, j}\right\}$ or $\left\{s_{i j}, s_{i, j+1}\right\}$ will be called a domino. A domino is removable from $Y_{p}$ if deleting its underlying squares leaves either another Young diagram containing the square $s_{11}$ or the empty set.

Successive deletions of removable dominos from a Young diagram $Y_{p}$ must eventually terminate in a staircase partition containing $\left(\begin{array}{c}r+1 \\ 2\end{array}\right)$ squares for some nonnegative integer $r$. This number is determined entirely by the underlying partition $p$ and does not depend on the sequence of deletions of removable dominos. We will write $p \in \mathcal{P}_{r}$, and we will say that $p$ is a partition of rank $r$. The core of $p$ is its underlying staircase partition.

EXAmple 3.1. The partition $p=\left[4,3^{2}, 1\right]$ lies in the set $\mathcal{P}_{2}$. Below are its Young diagram $Y_{p}$ and a domino tiling resulting from a sequence of deletions of removable dominos exhibiting the underlying staircase partition:
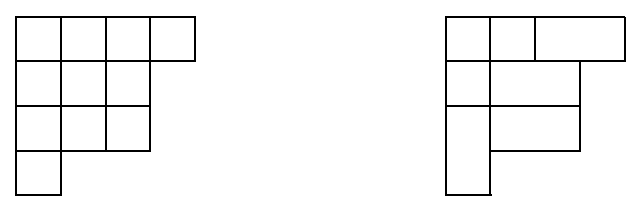

Consider $p \in \mathcal{P}_{r}$. It is a partition of the integer $2 n+\left(\begin{array}{c}r+1 \\ 2\end{array}\right)$ for some $n$. A standard domino tableau of rank $r$ and shape $p$ is a tiling of the noncore squares of $Y_{p}$ by dominos, each of which is labeled by a unique integer from $\{1, \ldots, n\}$ in such a way that the labels increase along its rows and columns. We will write $\operatorname{SDT}_{r}(p)$ for the set of standard domino tableaux of rank $r$ of shape $p$ and $\operatorname{SDT}_{r}(n)$ for the set of standard domino tableaux of rank $r$ which contain exactly $n$ dominos.

For $T \in \operatorname{SDT}_{r}(n)$, we will say that the square $s_{i j}$ is variable if $i+j \equiv$ $r \bmod 2$ and fixed otherwise. As discussed in [6] and [25], a choice of fixed squares on a tableau $T$ allows us to define two notions: a partition 
of its dominos into cycles and the operation of moving through a cycle. The moving-through map, when applied to a cycle $c$ in a tableau $T$, yields another standard domino tableau $M T(T, c)$, which differs from $T$ only in the labels of the variable squares of $c$. If $c$ contains $D(l, T)$, the domino in $T$ with label $l$, then $M T(T, c)$ is in some sense the minimally affected standard domino tableau in which the label of the variable square in $D(l, T)$ is changed (see [25] for detailed definitions).

If the shape of $M T(T, c)$ is the same as the shape of $T$, we will say that $c$ is a closed cycle. Otherwise, one square will be removed from $T$ (or added to its core), and one will be added. In this case, we will say the $c$ is open and denote the aforementioned squares as $s_{b}(c)$ and $s_{f}(c)$, respectively. Finally, if $s_{b}(c)$ is adjacent to the core of $T$, we will say that $c$ is a core open cycle. We will write $\mathrm{OC}(T)$ for the set of all open cycles of $T$ and $\mathrm{OC}^{*}(T)$ the subset of noncore open cycles.

\subsection{Generalized Robinson-Schensted algorithms}

The Weyl group $W_{n}$ of type $B_{n}$ consists of the set of signed permutations on $n$ letters, which we write in one-line notation as $w=\left(w_{1} w_{2} \cdots w_{n}\right)$. For each nonnegative integer $r$, there is an injective map

$$
G_{r}: W_{n} \rightarrow \operatorname{SDT}_{r}(n) \times \operatorname{SDT}_{r}(n)
$$

which is onto the subset of domino tableaux of the same shape (see [6] and [27]). We will write $G_{r}(x)=\left(S_{r}(x), T_{r}(x)\right)$ for the image of a permutation $x$, and we will refer to the two components as the left and right tableaux of $x$.

Definition 3.2. Consider $x, y \in W_{n}$, and fix a nonnegative integer $r$. We will say that

(1) $x \approx_{\mathcal{L}}^{\iota} y$ if $T_{r}(y)=T_{r}(x)$; and

(2) $x \approx_{\mathcal{L}} y$ if $T_{r}(y)=M T\left(T_{r}(x), C\right)$ for some $C \subset \mathrm{OC}^{*}\left(T_{r}(x)\right)$.

We will call the equivalence classes defined by $\approx_{\mathcal{L}}^{\iota}$ irreducible combinatorial left cells of rank $r$ in $W$, and those defined by $\approx_{\mathcal{L}}$ its reducible combinatorial left cells of rank $r$. In the irreducible case, we will say that the combinatorial left cell is represented by the tableau $T_{r}(x)$. In the reducible case, we will say that the combinatorial left cell is represented by the set $\left\{M T\left(T_{r}(x), C\right) \mid C \subset \mathrm{OC}^{*}\left(T_{r}(x)\right)\right\}$ of standard domino tableaux.

\subsection{Cells in type $B_{n}$}

Consider the generators of $W_{n}$ as in the following diagram: 


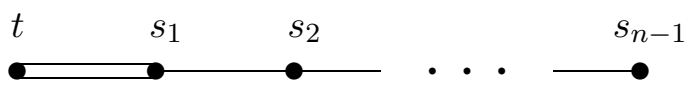

Define the weight function $L$ by $L(t)=b$ and $L\left(s_{i}\right)=a$ for all $i$, and set $s=b / a$. The following is a conjecture of Bonnafé, Geck, Iancu, and Lam and appears in $[3$, Conjectures $\mathrm{A}, \mathrm{B}$, and $\mathrm{D}]$.

Conjecture 3.3. Consider a Weyl group of type $B_{n}$ with a weight function $L$ and parameter $s$ defined as above.

(1) When $s \notin \mathbb{N}$, the Kazhdan-Lusztig left cells coincide with the irreducible combinatorial left cells of rank $\lfloor s\rfloor$.

(2) When $s \in \mathbb{N}$, the Kazhdan-Lusztig left cells coincide with the reducible combinatorial left cells of rank $s-1$.

This conjecture is well known to be true for $s=1$ by the work of Garfinkle [8] and has been verified when $s>n-1$ by Bonnafé and Iancu [4]. It has also been shown to hold for all values of $s$ when $n \leq 6$ (see [3]). Furthermore, assuming P1-P15, Bonnafé [2] has shown the conjecture to be true in the irreducible case and that, in the reducible case, Kazhdan-Lusztig left cells are unions of the reducible combinatorial left cells.

\section{$\S 4$. Constructible representations in type $B_{n}$}

Geck [9] has shown that if Lusztig's conjectures P1-P15 hold, then the representations of $W$ carried by the Kazhdan-Lusztig left cells of an unequalparameter Hecke algebra are precisely the constructible ones. Defined in the unequal-parameter setting by Lusztig [21, Section 20.15], constructible representations arise via truncated induction and tensoring with the sign representation. The goal of this section is to give a combinatorial description of the effects of these two operations on $W$-modules in type $B_{n}$. My approach is based on the equal-parameter results of [22].

\subsection{Irreducible $W_{n}$-modules}

We restrict our attention to type $B_{n}$; write $W_{n}$ for the corresponding Weyl group, and define constants $a, b$, and $s$ as in Section 3.3. Begin by recalling the standard parametrization of irreducible $W_{n}$-modules. Let $\mathcal{P}^{2}$ be the set of ordered pairs of partitions, and let $\mathcal{P}^{2}(n)$ be the subset of $\mathcal{P}^{2}$ where the combined sum of the parts of both partitions is $n$.

THEOREM 4.1. The set of irreducible representations of $W_{n}$ is parametrized by $\mathcal{P}^{2}(n)$. If we write $[(d, f)]$ for the representation corresponding to 
$(d, f) \in \mathcal{P}^{2}(n)$, then

$$
\left[\left(f^{t}, d^{t}\right)\right] \cong[(d, f)] \otimes \operatorname{sgn},
$$

where $p^{t}$ denotes the transpose of the partition $p$.

In this form, the connection between irreducible $W_{n}$-modules and the description of left cells in Conjecture 3.3 is not clear. To remedy this, we would like to restate Theorem 4.1 in terms of partitions of arbitrary rank which arise as shapes of the standard domino tableaux in this conjecture. Thus let $r=\lfloor s\rfloor$ if $s \notin \mathbb{N}$ and $r=s-1$ otherwise, and write $\epsilon=s-\lfloor s\rfloor$. As an intermediary to this goal, we define the notion of a symbol of defect $t$ and residue $\epsilon$ for a nonnegative integer $t$ and $0 \leq \epsilon<1$ as an array of nonnegative numbers of the form

$$
\Lambda=\left(\begin{array}{cccccc}
\lambda_{1}+\epsilon & \lambda_{2}+\epsilon & & \ldots & & \lambda_{N+t}+\epsilon \\
& \mu_{1} & \mu_{2} & \ldots & \mu_{N} &
\end{array}\right),
$$

where the (possibly empty) sequences $\left\{\lambda_{i}\right\}$ and $\left\{\mu_{i}\right\}$ consist of integers and are strictly increasing. If we define a related symbol by letting

$$
\Lambda^{\prime}=\left(\begin{array}{cccccc}
\epsilon & \lambda_{1}+1+\epsilon & \lambda_{2}+2+\epsilon & \ldots & & \lambda_{N+t}+N+t+\epsilon \\
0 & \mu_{1}+1 & \ldots & \mu_{N}+N &
\end{array}\right)
$$

then the binary relation defined by setting $\Lambda \sim \Lambda^{\prime}$ generates an equivalence relation. We will write $\mathrm{Sym}_{t}^{\epsilon}$ for the set of its equivalence classes.

We describe two maps between symbols and partitions. A partition can be used to construct a symbol in the following way. If $p=\left(p_{1}, p_{2}, \ldots, p_{k}\right)$, form $p^{\sharp}=\left(p_{1}, p_{2}, \ldots, p_{k^{\prime}}\right)$ by adding an additional zero term to $p$ if the rank of $p$ has the same parity as $k$. Dividing the set $\left\{p_{i}+k^{\prime}-i\right\}_{i=1}^{k^{\prime}}$ into its odd and even parts yields two sequences,

$$
\left\{2 \mu_{i}+1\right\}_{i=1}^{N}, \quad\left\{2 \lambda_{i}\right\}_{i=1}^{N+t},
$$

for some nonnegative integer $t$. A symbol $\Lambda_{p}$ of defect $t$ and residue $\epsilon$ corresponding to $p$ can now be defined by arranging the integers $\lambda_{i}$ and $\mu_{i}$ into an array as above.

Given a symbol of defect $t$ and residue $\epsilon$, it is also possible to construct an ordered pair of partitions. With $\Lambda$ as above, let

$$
d_{\Lambda}=\left\{\lambda_{i}-i+1\right\}_{i=1}^{N+t}, \quad f_{\Lambda}=\left\{\mu_{i}-i+1\right\}_{i=1}^{N} .
$$

Both constructions are well behaved with respect to the equivalence on symbols defined above. The next theorem follows from [16, Section 2.7]. 
TheOREM 4.2. The maps $p \mapsto \Lambda_{p}$ and $\Lambda \mapsto\left(d_{\Lambda}, f_{\Lambda}\right)$ define bijections

$$
\mathcal{P}_{r} \rightarrow \operatorname{Sym}_{r+1}^{\epsilon} \rightarrow \mathcal{P}^{2}
$$

for all values of $r$ and $\epsilon$. Consequently, their composition yields a bijection between $\mathcal{P}_{r}(n)$ and $\mathcal{P}^{2}(n)$.

This result allows us to custom tailor a parametrization of irreducible $W_{n}$-modules to each value of the parameter $s$ by defining $r$ and $\epsilon$ as above. Together with [21, Lemma 22.18], the present theorem implies the following alternate parametrization of the representations of $W_{n}$ in terms of symbols. A parametrization in terms of partitions of rank $r$ follows.

COROLlaRY 4.3. If we fix values of the defect $r$ and residue $\epsilon$, then the set of irreducible representations of $W_{n}$ is parametrized by the set of equivalence classes of symbols $\left\{\Lambda \in \operatorname{Sym}_{r+1}^{\epsilon} \mid\right.$ parts of $d_{\Lambda}$ and $f_{\Lambda}$ sum to $\left.n\right\}$. Writing $[\Lambda]$ for the representation corresponding to $\Lambda$, we have

$$
[\bar{\Lambda}]=[\Lambda] \otimes \operatorname{sgn}
$$

where the symbol $\bar{\Lambda}$ is defined from $\Lambda$ by the following procedure. Write $\Lambda$ as above, and let $\tau$ be the integer part of its largest entry. Then the integer parts of the top and bottom rows of $\bar{\Lambda}$ consist of the complements of $\left\{\tau-\mu_{i}\right\}_{i}$ and $\left\{\tau-\lambda_{i}\right\}_{i}$ in $[0, \tau] \cap \mathbb{Z}$, respectively.

COROLlaRY 4.4. If we fix a nonnegative integer $r$, then the set of irreducible representations of $W_{n}$ is parametrized by $\mathcal{P}_{r}(n)$. Writing $[p]$ for the representation corresponding to $p \in \mathcal{P}_{r}(n)$, we have

$$
\left[p^{t}\right] \cong[p] \otimes \operatorname{sgn},
$$

where $p^{t}$ is the transpose of the partition $p$.

EXAMPLE 4.5. Let $s=3 \frac{1}{2}$, so that $r=3$ and $\epsilon=1 / 2$, and consider the irreducible representation $\left[\left(\left(1^{3}\right),(1)\right)\right]$ of $W_{4}$. Then according to the above parametrizations, $\left[\left(\left(1^{3}\right),(1)\right)\right]=\left[\left(4,3,2^{2}\right)\right]=\left[\Lambda_{\left(4,3,2^{2}\right)}\right]$, where

$$
\Lambda_{\left[\left(4,3,2^{2}\right)\right]}=\left(\begin{array}{cccc}
\frac{1}{2} & 2 \frac{1}{2} & 3 \frac{1}{2} & 4 \frac{1}{2} \\
& & 1
\end{array}\right)
$$

is a symbol of defect 3 and residue $1 / 2$. Note that $\left(\left(1^{3}\right),(1)\right) \in \mathcal{P}^{2}(4)$, $\left(4,3,2^{2}\right) \in \mathcal{P}_{2}(4)$, and $\Lambda_{\left(4,3,2^{2}\right)}$ is a representative of a class in $\operatorname{Sym}_{3}^{\epsilon}$ for 
$\epsilon=1 / 2$. Furthermore, $\left[\left(\left(1^{3}\right),(1)\right)\right] \otimes \operatorname{sgn}=[((1),(3))]=\left[\left(4,3,2^{2}\right)\right] \otimes \operatorname{sgn}=$ $\left[\left(4^{2}, 2,1\right)\right]=\left[\Lambda_{\left(4,3,2^{2}\right)}\right] \otimes \operatorname{sgn}=\left[\Lambda_{\left(4^{2}, 2,1\right)}\right]$, where

$$
\Lambda_{\left[\left(4^{2}, 2,1\right)\right]}=\left(\begin{array}{cccc}
\frac{1}{2} & 1 \frac{1}{2} & 2 \frac{1}{2} & 4 \frac{1}{2} \\
& 3
\end{array}\right) .
$$

We will need the following lemma, which holds for finite $W$ whenever P1$\mathbf{P 1 5}$ hold. It is a combination of [21, Section 11.7] and [21, Section 21.5].

Lemma 4.6. Consider a Kazhdan-Lusztig left cell $\mathfrak{C} \subset W$, and let $w_{0}$ be the longest element of $W$. Then $\mathfrak{C} w_{0}$ is also a left cell in $W$, and $\left[\mathfrak{C} w_{0}\right] \cong \mathfrak{C} \otimes$ sgn as $W$-modules.

\subsection{Truncated induction}

We now turn to a combinatorial description of truncated induction in terms of the above parameter sets. If $\pi$ is a representation of $W_{I}$, a parabolic subgroup of $W_{n}$, Lusztig [21, Section 20.15] defined a representation $J_{W_{I}}^{W}(\pi)$ of $W=W_{n}$. Its precise definition depends on the parameters of the underlying Hecke algebra, so it is natural to expect that this is manifested in the combinatorics studied above. Following [22, Section 2] and [18], we note that, because of the transitivity of truncated induction and because the situation in type $A$ is well understood, we need only to understand how truncated induction works when $W_{I}$ is a maximal parabolic subgroup whose type $A$ component acts by the sign representation on $\pi$. Henceforth, let $W_{I}$ be a maximal parabolic subgroup in $W_{n}$ with factors $W^{\prime}$ of type $B_{m}$ and $S_{l}$ of type $A_{l-1}$, where $m+l=n$; furthermore, write $\operatorname{sgn}_{l}$ for the sign representation of $S_{l}$.

Truncated induction behaves well with respect to cell structure. In fact, the following lemma is true for general $W$ under the assumption that statements P1-P15 of Section 2.1 hold.

Lemma 4.7. ([10]) Let $\mathfrak{C}^{\prime}$ be a left cell of $W_{I}$. Then we have

$$
J_{W_{I}}^{W}\left(\left[\mathfrak{C}^{\prime}\right]\right) \cong[\mathfrak{C}]
$$

where $\mathfrak{C}$ is the left cell of $W$ such that $\mathfrak{C}^{\prime} \subset \mathfrak{C}$.

Let us first provide a description of the situation in type $B_{n}$ in terms of symbols. Consider a symbol $\Lambda^{\prime}$ of defect $r+1$ and residue $\epsilon$; via the equivalence on symbols, we can assume that it has at least $l$ entries. If the set of $l$ largest entries of $\Lambda^{\prime}$ is uniquely defined, then let $\Lambda$ be the symbol 
obtained by increasing each of the entries in this set by one. If it is not, then let $\Lambda^{\mathrm{I}}$ and $\Lambda^{\mathrm{II}}$ be the two symbols obtained by increasing the largest $l-1$ entries of $\Lambda^{\prime}$ and then each of the two $l$ th largest entries in turn by one.

Proposition 4.8. ([21]) The representation $J_{W_{I}}^{W}\left(\left[\Lambda^{\prime}\right] \otimes \operatorname{sgn}_{l}\right)$ is $[\Lambda]$ if the set of l largest entries of $\Lambda^{\prime}$ is uniquely defined, and $\left[\Lambda^{\mathrm{I}}\right]+\left[\Lambda^{\mathrm{II}}\right]$ if it is not. The former is always the case if $\left[\Lambda^{\prime}\right]$ is a symbol of residue $\epsilon \neq 0$.

It is not difficult to reformulate this result in terms of partitions of rank $r$. Consider a partition $p=\left(p_{1}, p_{2}, \ldots, p_{k}\right) \in \mathcal{P}_{r}$. We can assume that $k \geq l$ by adding zero parts to $p$ as necessary. Let $k^{\prime}$ be the number of parts of $p^{\sharp}$. Define

$$
\begin{aligned}
p^{\mathrm{I}} & =\left(p_{1}+2, \ldots, p_{l}+2, p_{l+1}, \ldots, p_{k}\right), \\
p^{\mathrm{II}} & =\left(p_{1}+2, \ldots, p_{l-1}+2, p_{l}+1, p_{l+1}+1, p_{r+2}, \ldots, p_{k}\right) .
\end{aligned}
$$

Note that both $p^{\mathrm{I}}$ and $p^{\mathrm{II}}$ are again partitions of rank $r$.

COROLlaRY 4.9. The representation $J_{W_{I}}^{W}\left([p] \otimes \operatorname{sgn}_{l}\right)$ produced by truncated induction is $\left[p^{\mathrm{I}}\right]$ whenever $p_{l}>p_{l+1}, p_{l}+r-l$ is odd, or $\epsilon \neq 0$. Otherwise,

$$
J_{W_{I}}^{W}\left([p] \otimes \operatorname{sgn}_{l}\right)=\left[p^{\mathrm{I}}\right]+\left[p^{\mathrm{II}}\right] .
$$

Proof. Using the results of Proposition 4.8, we have to check under what conditions the set of $l$ largest entries in a symbol $\Lambda^{\prime}$ is uniquely defined and then determine the preimages of the symbols $\Lambda^{\mathrm{I}}$ and $\Lambda^{\mathrm{II}}$ under the map of Theorem 4.2. When $\epsilon \neq 0$, the $l$ largest entries in $\Lambda^{\prime}$ are uniquely determined since all of its entries must be distinct. When $\epsilon=0$, there will be an ambiguity in determining the $l$ largest entries if and only if $p_{l}+k^{\prime}-l$ and $p_{l+1}+k^{\prime}-l-1$ are consecutive integers with the first one being odd. Together with the observation that $k^{\prime}$ is always of the opposite parity from $r$, this gives us the conditions of the proposition. Determining the partitions corresponding to $\Lambda^{\mathrm{I}}$ and $\Lambda^{\mathrm{II}}$ is then just a simple calculation.

Note that the parity conditions of the proposition imply that in the case when $J_{W_{I}}^{W}\left([p] \otimes \operatorname{sgn}_{l}\right)$ is reducible, the square $s_{l, p_{l}+1}$ of the Young diagrams of $p^{\mathrm{I}}$ and $p^{\mathrm{II}}$ is fixed. In particular, this means that, when endowed with the maximal label, the domino $\left\{s_{l, p_{l}+1}, s_{l, p_{l}+2}\right\}$ constitutes an open cycle in a domino tableau of shape $p^{\mathrm{I}}$. Its image under the moving-through map is $\left\{s_{l+1, p_{l}+1}, s_{l, p_{l}+1}\right\}$ with underlying partition $p^{\mathrm{II}}$. This observation leads to the following. 
LEMMA 4.10. Let $n=m+l$, and consider $w^{\prime}=\left(w_{1} w_{2} \cdots w_{m}\right) \in W_{m}$. Write $T^{\prime}=T_{r}\left(w^{\prime}\right)$ for its right tableau of rank $r$, and define a set of partitions

$$
\mathbb{P}^{\prime}=\left\{\text { shape } M T\left(T^{\prime}, C\right) \mid C \subset \mathrm{OC}^{*}\left(T^{\prime}\right)\right\} \subset \mathcal{P}_{r}(m)
$$

Define the set $\mathbb{P}=\left\{p^{\mathrm{I}} \mid p \in \mathbb{P}^{\prime}\right\} \cup\left\{p^{\mathrm{II}} \mid p \in \mathbb{P}^{\prime}\right.$ and $p_{l}=p_{l+1}$ with $p_{l}+r-l$ even $\}$. If $w=\left(w_{1} w_{2} \cdots w_{m} n n-1 \cdots m+1\right) \in W_{n}$ with right tableau $T=$ $T_{r}(w)$, then

$$
\mathbb{P}=\left\{\text { shape } M T(T, C) \mid C \subset \mathrm{OC}^{*}(T)\right\} \subset \mathcal{P}_{r}(n)
$$

Proof. The lemma relates the noncore open cycles in $T^{\prime}$ to the noncore open cycles in $T$; hence it follows from the description of the behavior of cycles under domino insertion in [25, Section 3.6]. However, things are really simpler than that. Note that $T$ is obtained from $T^{\prime}$ by placing horizontal dominos with labels $m+1$ through $n$ at the end of its first $l$ rows. Essentially, there are four possibilities. We write $s_{i j}$ for the left square of the domino added to row $i$, and we let $p=\operatorname{shape} T^{\prime}$.

(1) Here $s_{i j}=S_{f}(c)$ for a cycle $c$ of $T^{\prime}$. Then the domino joins the cycle $c$, and the final square of the new cycle is $s_{i, j+2}$.

(2) Here $s_{i j-1}=S_{b}(c)$ for a cycle $c$ of $T^{\prime}$. Then the domino joins the cycle $c$, and the beginning square of the new cycle is $s_{i, j+1}$.

(3) Here $p_{i-1}=p_{i}$ with $p_{i}+r-i$ odd. Then the dominos with labels $m+i-1$ and $m+i$ in $T$ form a closed cycle in $T$.

(4) Here $p_{l}=p_{l+1}$ with $p_{l}+r-l$ even. Then the domino with label $n$ forms a singleton noncore open cycle in $T$ that does not correspond to a cycle in $T^{\prime}$.

If $C \subset \mathrm{OC}^{*}\left(T^{\prime}\right)$ and $\widetilde{C}$ is the set of the corresponding cycles in $T$, then it is clear from the above description that $\left\{\right.$ shape $\left.M T(T, \widetilde{C}) \mid C \subset \mathrm{OC}^{*}\left(T^{\prime}\right)\right\}=$ $\left\{p^{\mathrm{I}} \mid p \in \mathbb{P}^{\prime}\right\}$. If case (4) arises and $T$ has an additional noncore open cycle $c=$ $\{n\}$, then $\left\{\right.$ shape $\left.M T(T, \widetilde{C} \cup c) \mid C \subset \mathrm{OC}^{*}\left(T^{\prime}\right)\right\}=\left\{p^{\mathrm{II}} \mid p \in \mathbb{P}^{\prime}\right\}$. The lemma follows.

EXAMPLE 4.11. Let $s=3$ so that $r=2$ and $\epsilon=0$, and consider the partition $\left(4,3,2^{3}\right) \in \mathcal{P}_{2}(5)$. It corresponds to the symbol

$$
\Lambda_{\left[\left(4,3,2^{3}\right)\right]}=\left(\begin{array}{cccc}
1 & 2 & 3 & 4 \\
& 1 &
\end{array}\right) \in \operatorname{Sym}_{3}^{0} .
$$


For $l=4$, we have $J_{W_{I}}^{W}\left(\left[\left(4,3,2^{3}\right)\right] \otimes \operatorname{sgn}_{4}\right)=\left[\left(6,5,4,3^{2}\right)\right]+\left[\left(6,5,4^{2}, 2\right)\right]$. Note that both partitions lie in $\mathcal{P}_{2}(9)$. In terms of symbols,

$$
J_{W_{I}}^{W}\left(\left[\Lambda_{\left(4,3,2^{3}\right)}\right] \otimes \operatorname{sgn}_{4}\right)=\left[\left(\begin{array}{cccc}
2 & 3 & 4 & 5 \\
& 1 &
\end{array}\right)\right]+\left[\left(\begin{array}{cccc}
1 & 3 & 4 & 5 \\
& 2 &
\end{array}\right)\right] .
$$

\section{$\S 5$. $W_{n}$-module structure and standard domino tableaux}

Viewing cells as constructible representations allows us to examine their structure inductively. Using the description of truncated induction and tensoring with sign derived in Section 4 , we describe the $W_{n}$-module carried by each cell in terms of the parametrization of irreducible $W_{n}$-modules of Section 4.1. First, we consider a few facts about combinatorial cells.

Lemma 5.1. Consider two combinatorial left cells $\mathfrak{C}$ and $\mathfrak{C}^{\prime}$ in $W_{n}$ of rank $r$ represented by sets $\mathbb{T}$ and $\mathbb{T}^{\prime}$ of rank $r$ standard domino tableaux. Then

$$
\left|\mathfrak{C} \cap \mathfrak{C}^{\prime-1}\right|=M,
$$

where $M$ is the number of tableaux in $\mathbb{T}$ whose shape matches the shape of a tableau in $\mathbb{T}^{\prime}$.

Proof. Suppose first that $\mathfrak{C}$ and $\mathfrak{C}^{\prime}$ are irreducible so that $\mathbb{T}=\{T\}$ and $\mathbb{T}^{\prime}=\left\{T^{\prime}\right\}$. If they are of the same shape, then the intersection $\mathfrak{C} \cap \mathfrak{C}^{\prime-1}=$ $G_{r}^{-1}\left(T^{\prime}, T\right)$; otherwise, it is empty.

On the other hand, if $\mathfrak{C}$ and $\mathfrak{C}^{\prime}$ are reducible, then let $J$ consist of the tableaux in $\mathbb{T}$ whose shapes match the shape of a tableau in $\mathbb{T}^{\prime}$, and define $|J|=M$. Recall that by the definition of a combinatorial left cell, $\mathbb{T}=\left\{M T(T, C) \mid C \in \mathrm{OC}^{*} T\right\}$ for some tableau $T$, and therefore $\mathbb{T}$ consists of only tableaux of differing shapes. If $T \in J$, write $T^{\prime}$ for the unique tableau in $\mathbb{T}^{\prime}$ of the same shape as $T$. Then

$$
\mathfrak{C} \cap \mathfrak{C}^{\prime-1}=\bigcup_{T \in J} G_{r}^{-1}\left(T^{\prime}, T\right) .
$$

We can obtain a slightly better description of the intersection of a combinatorial left cell and a combinatorial right cell by recalling the definition of an extended open cycle in a tableau relative to another tableau of the same shape (see [7, Definition 2.3.1] or [24, Definition 2.4] for details). In general, an extended open cycle is a union of open cycles. 
Corollary 5.2. Consider two reducible combinatorial left cells $\mathfrak{C}$ and $\mathfrak{C}^{\prime}$ in $W_{n}$ of rank $r$ represented by sets $\mathbb{T}$ and $\mathbb{T}^{\prime}$ of rank $r$ standard domino tableaux. If $T \in \mathbb{T}$ and $T^{\prime} \in \mathbb{T}^{\prime}$ are of the same shape and $m$ is the number of noncore extended open cycles in $T$ relative to $T^{\prime}$, then

$$
\left|\mathfrak{C} \cap \mathfrak{C}^{\prime-1}\right|=2^{m}
$$

Proof. An extended open cycle in $T$ relative to $T^{\prime}$ is a minimal set of open cycles in $T$ and $T^{\prime}$ such that moving through it produces another pair of tableaux of the same shape. Consequently, moving through two different extended open cycles are independent operations. Noting that

$$
\mathbb{T}=\left\{M T(T, C) \mid C \subset \mathrm{OC}^{*}(T)\right\}, \quad \mathbb{T}^{\prime}=\left\{M T\left(T^{\prime}, C\right) \mid C \subset \mathrm{OC}^{*}\left(T^{\prime}\right)\right\}
$$

we have that a tableau-pair $\left(S, S^{\prime}\right) \in \mathbb{T} \times \mathbb{T}^{\prime}$ is the same shape if and only if it differs from $\left(T, T^{\prime}\right)$ by moving through a set of noncore extended open cycles in $T$ relative to $T^{\prime}$. Thus, if $E$ is the set of noncore extended open cycles in $T$ relative to $T^{\prime}$, then

$$
\mathfrak{C} \cap \mathfrak{C}^{\prime-1}=\bigcup_{D \subset E} G_{r}^{-1}\left(M T\left(\left(T^{\prime}, T\right), D\right)\right)
$$

from which the result follows.

Recall the parameter $s$ derived from a weight function $L$ in type $B_{n}$. We will call a Kazhdan-Lusztig left cell in this setting a left cell of weight $s$. Bonnafé [2] has shown that

- under the assumption that statements P1-P15 of Section 2.1 hold, when $s \notin \mathbb{N}$, left cells of weight $s$ are precisely the irreducible combinatorial left cells of rank $r=\lfloor s\rfloor$, and

- when $s \in \mathbb{N}$, left cells of weight $s$ are unions of reducible combinatorial left cells of rank $r=s-1$.

In this way, as in Definition 3.2, we can say that a left cell of weight $s$ is represented by a set of standard domino tableaux of rank $r$. For noninteger $s$, this set consists of the unique tableau representing the irreducible combinatorial left cell, and in the latter, it is the union of the sets of tableaux representing each of the reducible combinatorial cells in the Kazhdan-Lusztig cell. In what follows, we assume that statements P1-P15 hold. 
Lemma 5.3. Suppose that $\mathfrak{C}$ is a left cell of weight $s$ and that $\mathfrak{C}=\coprod_{i} \mathfrak{D}_{i}$ is its decomposition into combinatorial left cells of rank $r$. If we let $\mathbb{T}_{i}$ be the set of domino tableaux representing $\mathfrak{D}_{i}$, then the set of shapes of tableaux in $\mathbb{T}_{i}$ is disjoint from the set of shapes of tableaux in $\mathbb{T}_{j}$ whenever $i \neq j$.

Proof. By Corollary 2.5, $\mathfrak{C} \cap \mathfrak{C}^{-1}$ consists of the involutions in $\mathfrak{C}$. The set of involutions in each combinatorial cell $\mathfrak{D}_{i}$ consists of $\mathfrak{D}_{i} \cap \mathfrak{D}_{i}^{-1}$. This forces $\mathfrak{D}_{i} \cap \mathfrak{D}_{j}^{-1}=\varnothing$ whenever $i \neq j$, which can occur only if the set of shapes of tableaux in $\mathbb{T}_{i}$ is disjoint from the set of shapes of tableaux in $\mathbb{T}_{j}$ by Lemma 5.1 .

Let us first show that the shapes of the standard domino tableaux of rank $r$ representing a left cell of weight $s$ determine its $W_{n}$-module structure.

Definition 5.4. Suppose that $\mathbb{T}$ is a set of standard domino tableaux of rank $r$. For $T \in \mathbb{T}$, write $p_{T} \in \mathcal{P}_{r}(n)$ for its underlying partition, and define

$$
[\mathbb{T}]=\bigoplus_{T \in \mathbb{T}}\left[p_{T}\right]
$$

Lemma 5.5. Suppose that $\mathfrak{C}$ and $\mathfrak{C}^{\prime}$ are left cells of weight $s$ in $W_{n}$, and suppose that

$$
\mathfrak{C}=\coprod_{i \leq c} \mathfrak{D}_{i}, \quad \mathfrak{C}^{\prime}=\coprod_{i \leq d} \mathfrak{D}_{i}^{\prime}
$$

are their decompositions into combinatorial left cells of rank $r$. Suppose that each $\mathfrak{D}_{i}$ and $\mathfrak{D}_{i}^{\prime}$ is represented by the set of rank $r$ tableaux $\mathbb{T}_{i}$ and $\mathbb{T}_{i}^{\prime}$, respectively. Then $[\mathfrak{C}] \cong\left[\mathfrak{C}^{\prime}\right]$ if and only if $c=d$ and, suitably ordered, $\left[\mathbb{T}_{i}\right] \cong$ $\left[\mathbb{T}_{i}^{\prime}\right]$ for all $i$.

Proof. For clarity, let us treat the integer and noninteger values of $s$ separately. First, assume that $s \notin \mathbb{N}$ so that $c=d=1$, and take $\{T\}=\mathbb{T}_{1}$ and $\left\{T^{\prime}\right\}=\mathbb{T}_{1}^{\prime}$. By Lemmas 2.4 and 5.1, we have $\operatorname{dim} \operatorname{Hom}_{W}([\mathfrak{C}],[\mathfrak{C}])=$ $\operatorname{dim} \operatorname{Hom}_{W}\left(\left[\mathfrak{C}^{\prime}\right],\left[\mathfrak{C}^{\prime}\right]\right)=1$. Furthermore, we have $\operatorname{dim} \operatorname{Hom}_{W}\left(\left[\mathfrak{C}^{\mathfrak{C}}\right],\left[\mathfrak{C}^{\prime}\right]\right)=\mid \mathfrak{C} \cap$ $\mathfrak{C}^{\prime-1} \mid=1$ if and only if the shapes of $T$ and $T^{\prime}$ coincide; otherwise, $\operatorname{dim} \operatorname{Hom}_{W}\left([\mathfrak{C}],\left[\mathfrak{C}^{\prime}\right]\right)=0$. Lemma 5.5 follows.

Next, assume that $s \in \mathbb{N}$. Suppose first that $[\mathfrak{C}] \cong\left[\mathfrak{C}^{\prime}\right]$. Then $\operatorname{dim} \operatorname{Hom}(\mathfrak{C}$, $\mathfrak{C})=\operatorname{dim} \operatorname{Hom}\left(\mathfrak{C}^{\prime}, \mathfrak{C}^{\prime}\right)=\operatorname{dim} \operatorname{Hom}\left(\mathfrak{C}, \mathfrak{C}^{\prime}\right)$, and by Lemma 2.4, $\left|\mathfrak{C}^{\prime} \cap \mathfrak{C}^{-1}\right|=$ $\left|\mathfrak{C}^{\prime} \cap \mathfrak{C}^{\prime-1}\right|=\left|\mathfrak{C} \cap \mathfrak{C}^{-1}\right|$. By Lemma 5.3, we have

$$
\sum_{i \leq c}\left|\mathfrak{D}_{i} \cap \mathfrak{D}_{i}^{-1}\right|=\sum_{i \leq d}\left|\mathfrak{D}_{i}^{\prime} \cap \mathfrak{D}_{i}^{\prime-1}\right|=\sum_{i, j}\left|\mathfrak{D}_{i} \cap \mathfrak{D}_{j}^{\prime-1}\right| .
$$


We can now use Corollary 5.2 to examine the terms of this equality. For a combinatorial cell $\mathfrak{D}_{i}$, there is at most one cell $\mathfrak{D}_{i^{\prime}}^{\prime}$ such that there are $T_{i} \in \mathbb{T}_{i}$ and $T_{i^{\prime}}^{\prime} \in \mathbb{T}_{i^{\prime}}^{\prime}$ of the same shape, by Lemma 5.3. Let $I$ be the set of $i$ for which this occurs. Let $c_{i}$ and $d_{i}$ be the numbers of noncore open cycles in $T_{i}$ and $T_{i^{\prime}}^{\prime}$, and for each $i \in I$, let $m_{i}$ be the number of noncore extended open cycles in $T_{i}$ relative to $T_{i^{\prime}}^{\prime}$. Then $m_{i} \leq c_{i}, d_{i^{\prime}}$ with equality if and only if the noncore extended open cycles are just the noncore open cycles. By Corollary 5.2, $\sum_{i \leq c}\left|\mathfrak{D}_{i} \cap \mathfrak{D}_{i}^{-1}\right|=\sum_{i \leq c} 2^{c_{i}}, \sum_{i \leq d}\left|\mathfrak{D}_{i}^{\prime} \cap \mathfrak{D}_{i}^{\prime-1}\right|=$ $\sum_{i \leq d} 2^{d_{i}}$, and $\sum_{I}\left|\mathfrak{D}_{i} \cap \mathfrak{D}_{i^{\prime}}^{\prime-1}\right|=\sum_{I} 2^{m_{i}}$. But the previous equation now implies that $m_{i}=c_{i}=d_{i^{\prime}}, c=d, I=\{1, \ldots, c\}$, and, by the definition of a combinatorial left cell in our setting, $\left[\mathbb{T}_{i}\right] \cong\left[\mathbb{T}_{i^{\prime}}^{\prime}\right]$ for all $i \in I$.

Conversely, assume that $c=d$ and $\left[\mathbb{T}_{i}\right] \cong\left[\mathbb{T}_{i}^{\prime}\right]$ for all $i$, and choose tableaux $T_{i} \in \mathbb{T}_{i}$ and $T_{i}^{\prime} \in \mathbb{T}_{i}^{\prime}$ of the same shape. By the definition of combinatorial cells, there is a correspondence between the noncore open cycles of $T_{i}$ and those of $T_{i}^{\prime}$ such that their beginning and final squares coincide, implying that the set of noncore extended open cycles in $T_{i}$ relative to $T_{i}^{\prime}$ is precisely the set of open cycles of $T_{i}$. Therefore, for each $i$ we have $\left|\mathfrak{D}_{i} \cap \mathfrak{D}_{i}^{-1}\right|=$ $\left|\mathfrak{D}_{i} \cap \mathfrak{D}_{i}^{\prime-1}\right|$. Consequently, by Lemmas 5.3 and 2.4 and Corollary 5.2,

$$
\operatorname{dim} \operatorname{Hom}\left(\mathfrak{C}, \mathfrak{C}^{\prime}\right)=\sum_{i}\left|\mathfrak{D}_{i} \cap \mathfrak{D}_{i}^{\prime-1}\right|=\sum_{i}\left|\mathfrak{D}_{i} \cap \mathfrak{D}_{i}^{-1}\right|=\operatorname{dim} \operatorname{Hom}(\mathfrak{C}, \mathfrak{C})
$$

Reversing the roles of $\mathfrak{C}$ and $\mathfrak{C}^{\prime}$ above implies the desired result.

TheOREM 5.6. Suppose that $\mathfrak{C}$ is a left cell of weight $s$ in $W_{n}$ represented by a set $\mathbb{T}$ of standard domino tableaux of rank $r$. Then $[\mathfrak{C}] \cong[\mathbb{T}]$ as $W_{n^{-}}$ modules.

Proof. In light of the result from Lemma 5.5, we can prove the theorem by verifying that it holds for a representative of each isomorphism class of left cells. Under our assumptions, the results of [9] hold, and left cell modules coincide with constructible representations of $W_{n}$. Therefore, a representative of each isomorphism class of left cells can be obtained by repeated truncated induction and tensoring with sign. Recall the description of irreducible $W_{n}$-modules by partitions of rank $r$. Via Corollaries 4.4 and 4.9 , we have a description of both operations on the level of partitions. We verify that the effect of truncated induction and tensoring with sign on the shapes of the tableaux representing a left cell is the same, and the theorem follows by induction. 
Let us treat the integer and noninteger values of $s$ separately. First, assume that $s \notin \mathbb{N}$, so that each left cell is represented by a single tableau. Begin by investigating the effect on tensoring with sign. If $[\mathfrak{C}]$ is a left cell module and $w \in \mathfrak{C}$, then $\mathfrak{C}$ is represented by the tableau $T_{r}(w)$ of shape $p$. By Lemma $4.6, \mathfrak{C} w_{0}$ is also a left cell, and $\left[\mathfrak{C} w_{0}\right] \cong[\mathfrak{C}] \otimes \operatorname{sgn}$. It is represented by the tableau $T_{r}\left(w w_{0}\right)=T_{r}(w)^{t}$ of shape $p^{t}$. By Corollary 4.4 , if we assume that $[\mathfrak{C}]$ carries the irreducible module associated to the shape of its representative tableau, then so does $\left[\mathfrak{C} w_{0}\right] \cong[\mathfrak{C}] \otimes$ sgn.

For the case of truncated induction, consider a maximal parabolic subgroup $W_{I}=W_{m} \times S_{l}$ of $W_{n}$. Choose $w^{\prime}=\left(w_{1} w_{2} \cdots w_{m}\right) \in W_{m}$, and let $\mathfrak{C}^{\prime}$ be its left cell, represented by the tableau $T^{\prime}=T_{r}\left(w^{\prime}\right)$. Let $p=\operatorname{shape} T^{\prime}$. By Lemma 4.7, $J_{W_{I}}^{W}\left(\left[\mathfrak{C}^{\prime}\right] \otimes \operatorname{sgn}_{l}\right)=[\mathfrak{C}]$ for a left cell $\mathfrak{C} \subset W_{n}$, and furthermore, the element $w=\left(w_{1} w_{2} \cdots w_{m} n n-1 \cdots m+1\right) \in \mathfrak{C}$. Using the notation of Corollary 4.9 , the left cell $\mathfrak{C}$ is represented by the tableau $T_{r}(w)$ whose shape is $p^{I}$. By Corollary 4.9 , if we assume that $\left[\mathfrak{C}^{\prime}\right]$ carries the irreducible module associated to the shape of its representative tableau, then so does $[\mathfrak{C}] \cong J_{W_{I}}^{W}\left(\left[\mathfrak{C}^{\prime}\right] \otimes \operatorname{sgn}_{l}\right)$.

Next assume that $s \in \mathbb{N}$, so that each left cell is represented by a family of rank $r$ standard domino tableaux. Again, we begin by investigating the effect on tensoring with sign. Suppose that $\mathfrak{C}$ is a left cell represented by the set $\mathbb{T}$, and suppose that, for each $T \in \mathbb{T}, w_{T} \in W_{n}$ is chosen so that $T_{r}\left(w_{T}\right)=T$. By Lemma 4.6, $\mathfrak{C} w_{0}$ is also a left cell, and $\left[\mathfrak{C} w_{0}\right] \cong[\mathfrak{C}] \otimes$ sgn. It is represented by the set of tableaux $T_{r}\left(w_{T} w_{0}\right)=T_{r}\left(w_{T}\right)^{t}($ for $T \in \mathbb{T})$, which we write as $\mathbb{T}^{t}$. By Corollary 4.4 , if we assume that $[\mathfrak{C}]$ carries the module $[\mathbb{T}]$, then $\left[\mathfrak{C} w_{0}\right] \cong[\mathfrak{C}] \otimes$ sgn carries the module $\left[\mathbb{T}^{t}\right]$.

For the case of truncated induction, again consider a maximal parabolic subgroup $W_{I}=W_{m} \times S_{l}$ of $W_{n}$. Let $\mathfrak{C}^{\prime}$ be a left cell of $W_{m}$, and let $\mathfrak{C}^{\prime}=\coprod_{i} \mathfrak{D}_{i}^{\prime}$ be its decomposition into combinatorial left cells. Suppose that $\mathfrak{D}_{i}^{\prime}$ is represented by the set $\mathbb{T}_{i}^{\prime}$ of domino tableaux, and let $\mathbb{T}^{\prime}=\coprod_{i} \mathbb{T}_{i}^{\prime}$. By definition of combinatorial left cells, every $\mathbb{T}_{i}^{\prime}=\left\{M T\left(T_{i}^{\prime}, C\right) \mid C \subset \mathrm{OC}^{*}\left(T_{i}^{\prime}\right)\right\}$ for some rank $r$ standard domino tableau $T_{i}^{\prime}$. For each $i$, choose $\widetilde{w}^{i}=\left(w_{1}^{i} w_{2}^{i} \cdots w_{m}^{i}\right) \in$ $W_{m}$ with $T_{i}^{\prime}=T_{r}\left(\widetilde{w}^{i}\right)$ so that $\widetilde{w}^{i} \in \mathfrak{D}_{i}^{\prime}$. By Lemma 4.7, $J_{W_{I}}^{W}\left(\left[\mathfrak{C}^{\prime}\right] \otimes \operatorname{sgn}_{l}\right)=$ $[\mathfrak{C}]$ for a left cell $\mathfrak{C} \subset W_{n}$. Furthermore, $w^{i}=\left(w_{1}^{i} w_{2}^{i} \cdots w_{m}^{i} n n-1 \cdots m+\right.$ $1) \in \mathfrak{C}$, and if $T_{i}=T_{r}\left(w^{i}\right)$, then $\mathfrak{C}$ is represented by the set of tableaux $\mathbb{T}=\coprod_{i}\left\{M T\left(T_{i}, C\right) \mid C \subset \mathrm{OC}^{*}\left(T_{i}\right)\right\}$. Lemma 4.10 describes the shapes of the tableaux in $\mathbb{T}$ in terms of the shapes of the tableaux in $\mathbb{T}^{\prime}$. This, together with Corollary 4.9, shows that if we assume that $\left[\mathfrak{C}^{\prime}\right]$ carries the module $\left[\mathbb{T}^{\prime}\right]$, then $[\mathfrak{C}] \cong J_{W_{I}}^{W}\left(\left[\mathfrak{C}^{\prime}\right] \otimes \operatorname{sgn}_{l}\right)$ carries the module $[\mathbb{T}]$. 
Corollary 5.7. Consider a Weyl group of type $B_{n}$ with a weight function $L$ and parameter $s$ defined as above. If statements $\mathbf{P 1 - P 1 5}$ hold, then

(1) when $s \notin \mathbb{N}$, the Kazhdan-Lusztig left cells of weight s coincide with the irreducible combinatorial left cells of rank $\lfloor s\rfloor$, and

(2) when $s \in \mathbb{N}$, the Kazhdan-Lusztig left cells of weight s coincide with the reducible combinatorial left cells of rank $s-1$.

If the set $\mathbb{T}$ of standard domino tableaux represents the left cell $\mathfrak{C}$ in $W_{n}$, then $[\mathfrak{C}] \cong[\mathbb{T}]$ as $W_{n}$-modules. Furthermore, if $T \in \mathbb{T}$, then the number of elements of $\mathfrak{C}$ with right tableau $T$ is the dimension of the irreducible constituent $\left[p_{T}\right]$ of $[\mathfrak{C}]$.

Proof. The first part in the case $s \notin \mathbb{N}$ is a result of Bonnafé [2]. To verify it in the case $s \in \mathbb{N}$, write a Kazhdan-Lusztig left cell $\mathfrak{C}$ in terms of combinatorial left cells as $\mathfrak{C}=\coprod_{i \in I} \mathfrak{D}_{i}$. Since $[\mathfrak{C}]$ is constructible, the main result of $[23]$ shows that $[\mathfrak{C}] \cong[\widetilde{\mathbb{T}}]$ as $W_{n}$-modules where $\widetilde{\mathbb{T}}=\{M T(T, C) \mid$ $\left.C \subset \mathrm{OC}^{*}(T)\right\}$ for some standard domino tableau $T$ of rank $r$. Let each $\mathfrak{D}_{i}$ be represented by $\mathbb{T}_{i}=\left\{M T\left(T_{i}, C\right) \mid C \subset \mathrm{OC}^{*}\left(T_{i}\right)\right\}$, and write $\mathbb{T}=\coprod_{i \in I} \mathbb{T}_{i}$. By Theorem 5.6, $[\mathbb{T}]=[\widetilde{\mathbb{T}}]$. This implies that for every $i$, the set of beginning and ending squares of noncore open cycles in $T_{i}$ is contained in the corresponding set in $T$. However, the size of this set is constant for every partition in the set of possible shapes of tableaux in $[\mathbb{T}]$. By Lemma 5.3, the only way this can occur is if $|I|=1$, that is, if $\mathfrak{C}$ consists of a single combinatorial cell.

Finally, we verify the last statement of the corollary. If $s \notin \mathbb{N}$, consider a left cell $\mathfrak{C}$ represented by the tableau $T$. Then $\operatorname{dim}[\mathfrak{C}]=\sum\left|\mathfrak{C} \cap \mathfrak{C}^{\prime-1}\right|$, the sum taken over all left cells $\mathfrak{C}^{\prime}$ in $W_{n}$. But $\left|\mathfrak{C} \cap \mathfrak{C}^{\prime-1}\right|=1$ if and only if the shapes of the tableaux representing $\mathfrak{C}$ and $\mathfrak{C}^{\prime}$ are the same; otherwise it is zero. Since each left cell is represented by a unique tableau, the above sum equals the number of tableaux of the same shape as $T$. This is the same as the number of elements of $\mathfrak{C}$ with right tableau $T$. If $s \in \mathbb{N}$, consider left cells $\mathfrak{C}$ and $\mathfrak{C}^{\prime}$. For $w \in \mathfrak{C} \cap \mathfrak{C}^{\prime-1}$, [ $\left.\operatorname{shape} T_{r}(w)\right]$ must be a component of both $[\mathfrak{C}]$ and $\left[\mathfrak{C}^{\prime}\right]$. Furthermore, each $w \in \mathfrak{C} \cap \mathfrak{C}^{\prime-1}$ must have the right tableau of a unique shape, establishing a bijection between $\mathfrak{C} \cap \mathfrak{C}^{\prime-1}$ and the set of irreducible modules common to $[\mathfrak{C}]$ and $\left[\mathfrak{C}^{\prime}\right]$. If we let $\mathfrak{C}^{\prime}$ vary over all left cells of $W_{n}$, the statement follows by Lemma 2.4 .

It should be remarked that the above statement classifying the module structure of left cells is not the strongest one could hope for. In the so-called "asymptotic" case when $s$ is sufficiently large, Geck [12, Section 5] has 
shown that, whenever the tableaux representing $[\mathfrak{C}]$ and $\left[\mathfrak{C}^{\prime}\right]$ are equal, not only are the underlying $\mathcal{H}$-modules isomorphic, but the underlying structure constants are also the same. More precisely, there is a bijection $\mathfrak{C} \rightarrow \mathfrak{C}^{\prime}$ sending $x \mapsto x^{\prime}$ such that

$$
h_{w, x, y}=h_{w, x^{\prime}, y^{\prime}}, \quad \text { for all } w \in W_{n} \text { and } x, y \in \mathfrak{C}
$$

It would be interesting to know under what circumstances this stronger statement holds for other values of $s$.

\section{REFERENCES}

[1] D. Alvis, The left cells of the Coxeter group of type $H_{4}$, J. Algebra 107 (1987), $160-168$.

[2] C. Bonnafé, On Kazhdan-Lusztig cells in type B, preprint, arXiv:0806.0214v2 [math.RT]

[3] C. Bonnafé, M. Geck, L. Iancu, and T. Lam, On domino insertion and KazhdanLusztig cells in type $B_{n}$, to appear in Progr. Math., preprint.

[4] C. Bonnafé and L. Iancu, Left cells in type $B_{n}$ with unequal parameters, Represent. Theory 7 (2003), 587-609.

[5] F. DuCloux, Positivity results for the Hecke algebras of noncrystallographic finite Coxeter group, J. Algebra 303 (2006), 731-741.

[6] D. Garfinkle, On the classification of primitive ideals for complex classical Lie algebras (I), Compos. Math. 75 (1990), 135-169.

[7] D. Garfinkle, On the classification of primitive ideals for complex classical Lie algebras (II), Compos. Math. 81 (1992), 307-336.

[8] D. Garfinkle, On the classification of primitive ideals for complex classical Lie algebras (III), Compos. Math. 88 (1993), 187-234.

[9] M. Geck, Constructible characters, leading coefficients and left cells for finite Coxeter groups with unequal parameters, Represent. Theory 6 (2002), 1-30.

[10] M. Geck, Left cells and constructible representations, Represent. Theory 9 (2005), $385-416$.

[11] M. Geck, Kazhdan-Lusztig cells and the Murphy basis, Proc. Lond. Math. Soc. 93 (2006), 635-665.

[12] M. Geck, Relative Kazhdan-Lusztig cells, Represent. Theory 10 (2006), 481-524.

[13] M. Geck, On Iwahori-Hecke algebras with unequal parameters and Lusztig's isomorphism theorem, to appear in Pure Appl. Math. Q., preprint.

[14] M. Geck and L. Iancu, Lusztig's a-function in type $B_{n}$ in the asymptotic case, Nagoya Math. J. 182 (2006), 199-240.

[15] M. Geck and G. Pfeiffer, Characters of Finite Coxeter Groups and Iwahori-Hecke Algebras, Lond. Math. Soc. Monogr. N.S. 21, Oxford University Press, New York, 2000.

[16] G. James and A. Kerber, The Representation Theory of the Symmetric Group, Encyclopedia Math. Appl. 16, Addison-Wesley, Reading, MA, 1981.

[17] D. A. Kazhdan and G. Lusztig, Schubert varieties and Poincaré duality, Proc. Sympos. Pure Math. 34 (1980), 185-203. 
[18] G. Lusztig, A class of irreducible representations of a Weyl group II, Indag. Math. 44 (1982), 219-226.

[19] G. Lusztig, Characters of Reductive Groups over a Finite Field, Ann. of Math. Stud. 207, Princeton University Press, Princeton, 1984.

[20] G. Lusztig, "Left cells in Weyl groups," in Lie Group Representations, Lecture Notes in Math. 1024, Springer, Berlin, 1983, 99-111.

[21] G. Lusztig, Hecke Algebras with Unequal Parameters, CRM Monogr. Ser. 18, Am. Math. Soc., Providence, 2003.

[22] W. M. McGovern, Left cells and domino tableaux in classical Weyl groups, Compos. Math. 101 (1996), 77-98; see also Errata, Compos. Math. 117 (1999), 117-121.

[23] T. Pietraho, Cells and constructible representations in type $B_{n}$, New York J. Math. 14 (2008), 411-430.

[24] T. Pietraho, Equivalence classes in the Weyl groups of type $B_{n}$, J. Algebraic Combin. 27 (2008), 247-262.

[25] T. Pietraho, A relation for domino Robinson-Schensted algorithms, Ann. Comb., 13 (2010), 519-532.

[26] T. A. Springer, Quelques applications de la cohomologie d'intersection, Sémin. Bourbaki 589 (1982), 249-273.

[27] M. A. A. van Leeuwen, The Robinson-Schensted and Schutzenberger algorithms, an elementary approach, Electron. J. Combin. 3 (1996).

Department of Mathematics

Bowdoin College

Brunswick, Maine 04011

$U S A$

tpietrah@bowdoin. edu 